\title{
Emotions associated with different textures during touch
}

\author{
Marina Iosifyan ${ }^{1,2}$, Olga Korolkova ${ }^{3,4}$ \\ ${ }^{1}$ - Moscow State University, Faculty of Psychology, 11/9 Mokhovaya Street, 125009, Moscow, \\ Russia \\ 2 - National Research University Higher School of Economics, 20 Myasnitskaya Street, 101000, \\ Moscow, Russia \\ 3 - Brunel University London, College of Health and Life Sciences, Department of Life Sciences, \\ Kingston Lane, Uxbridge, Middlesex, UB8 3PH, United Kingdom \\ ${ }^{4}$ - Moscow State University of Psychology and Education, Centre for Experimental Psychology, 2a \\ Shelepikhinskaya Quay, 123290, Moscow, Russia
}

Corresponding author: Marina Iosifyan, marina.iosifyan@gmail.com, Moscow State University, Faculty of Psychology, 11/9 Mokhovaya Street, 125009, Moscow, Russia. 


\section{Emotions associated with different textures during touch}

Haptics plays an important role in emotion perception. However, most studies of the affective aspects of haptics have investigated emotional valence rather than emotional categories. In the present study, we explored the associations of different textures with six basic emotions: fear, anger, happiness, disgust, sadness and surprise. Participants touched twenty-one different textures and evaluated them using six emotional scales. Additionally, we explored whether individual differences in participants' levels of alexithymia are related to the intensity of emotions associated with touching the textures. Alexithymia is a trait related to difficulties in identifying, describing and communicating emotions to others. The findings show that people associated touching different textures with distinct emotions. Textures associated with each of the basic emotions were identified. The study also revealed that a higher alexithymia level corresponds to a higher intensity of associations between textures and the emotions of disgust, anger and sadness.

Keywords: haptics; emotion; touch; emotion intensity; alexithymia

- Most studies of the emotional aspects of touch explored the pleasantness of touching

- In this study, we explored how touching textures is associated with basic emotions

- We found that people associate distinct emotions with textures while touching them 


\section{Introduction}

The haptic modality provides a substantial contribution to generating and modifying emotions (Gallace \& Spence, 2010). We extensively use haptics in social communication when we want to express gratitude, dominance or support (Eibl-Eibesfeldt, 1989; Suvilehto et al., 2015). Social affective touch, such as consoling touch, has both neural and behavioral specificities (Peled-Avron et al., 2017). Touch communication between mothers and infants, as well as the petting of animals, is related to reduced stress (Feldman et al., 2010; Jenkins, 1986; Lass-Hennemann et al., 2014; Stack \& Muir, 1990). Touching some inanimate objects and textures can also be associated with pleasant feelings, while touching others induces unpleasant ones (Etzi et al., 2014, 2016).

The importance of conveying different aspects of emotions through haptics has been recognized in an increasing number of studies (see Gallace \& Spence, 2010). Most studies investigating affective haptics have explored how touch can communicate a positive or negative valence of emotions (Ackerley et al., 2014; Croy et al., 2016; Essick et al., 2010; Löken et al., 2009), as well as the parameters of a stimulus that contribute to the pleasantness of touch (Essick et al., 2010). In particular, empirical studies have shown that a texture's softness and smoothness correlate with the pleasantness of touching it (Ekman et al., 1965; Major, 1895; Verrillo et al., 1999). Other studies have investigated unpleasantness and negative affects in touch, such as painful sensations (Fernandez \& Turk, 1992; Field, 2010). Unpleasant sensations of touch have been widely investigated in relation to individual differences, such as autism spectrum disorders (Cascio et al., 2008; Jones et al., 2009).

Despite the large number of studies exploring valence in touch, less focus has been given to the perception of distinct emotions (such as anger, fear or happiness) in haptics. Researchers have shown that people can communicate and accurately decode such emotions as anger, fear, disgust, love, gratitude and sympathy via social touch (Hertenstein et al., 2006; 2009; Kirsch et al., 2018). Other 
studies have shown that tactile surfaces are associated with words related to emotional states (Etzi et al., 2016; Iosifyan, Korolkova \& Vlasov, 2017). In the current study, we investigated whether different textures might be associated with six basic emotions: fear, anger, happiness, disgust, sadness and surprise (Ekman, 1993). These emotions can be readily recognized across other modalities, such as vision and audio, via facial expressions (Ekman, 1993), body posture (Van den Stock, Righart, \& de Gelder, 2007), emotional speech (de Gelder \& Vroomen, 2000), non-verbal vocalizations (Sauter, Eisner, Ekman, \& Scott, 2010) and music (Mohn, Argstatter, \& Wilker, 2011); to our knowledge, however, there have been no studies of the possible association of basic emotions and textures experienced through touch.

Studies of emotion processing in the visual and auditory modalities have revealed that individual differences can play a substantial role in emotion perception (Hamann \& Canli, 2004; Martins et al., 2017). In the present study, we focused on individual differences in alexithymia levels. Alexithymia is a personality construct based on difficulties in emotion processing and communicating emotions to others (McDonald \& Prkachin, 1990; Taylor, Bagby \& Parker, 1997). People with high levels of alexithymia have more difficulty in recognizing emotional expressions (Lane et al., 1996; Parker, Prkachin \& Prkachin, 2005). In particular, they demonstrate diminished perception of facial expressions of some — but not all — negative emotions: sadness, anger and fear (Prkachin, Casey \& Prkachin, 2009). There are also differences in the perception of emotion intensity that are related to alexithymia (Cecchetto, Rumiati \& Aiello, 2017). However, the majority of these findings connect alexithymia to the visual, auditory and olfactory modalities, and little is known about the processing of emotions conveyed by tactile stimuli in alexithymia. In the current study, we tested the hypothesis that a person's alexithymia level is related to emotion intensity in the haptic modality. 
In the current study we aimed to answer several questions: 1) Are textures associated with the basic emotions when they are touched? 2) Does the intensity of these emotions differ? 3) Is a person’s alexithymia level related to the perceived emotion intensity?

We used a paradigm of active touch (participants actively explore textures with their palms and fingers) instead of passive touch (a texture is moved against the participant's skin). Firstly, on the behavioral level, active touch allows for a more selective and controlled way of encoding tactile stimulus properties (Chapman, 1994; Lederman \& Klatzky, 1987). Secondly, on the neural level, active touch elicits greater activation in the primary somatosensory region and does not differ from passive touch in the secondary somatosensory region (Simoes-Franklin, Whitaker \& Newell, 2011). Thirdly, we supposed that active exploration of tactile stimuli is a more ecological task, close to real life experience, compared to passive touch wherein a participant does not perform any actions.

\section{Method}

\subsection{Participants}

One hundred and eight participants took part in the study (age range: 18-47 years, $M_{\text {age }}=$ 21.37, $S D=4.37,22$ males). Participants were recruited at the Lomonosov University campus and received course credit in return for their participation. Participants had normal tactile sensitivity by self-report and no mental disorders. Six of them were left-handed by self-report and performed the study task with their left hands, while others were right-handed. Participants gave informed written consent prior to taking part in the study. The study was carried out in accordance with The Code of Ethics of the World Medical Association (Declaration of Helsinki). It was approved by the Ethical Committee of the Institute of Psychology, Russian Academy of Sciences.

\subsection{Materials}


Twenty-one different tactile surfaces were used as stimuli. Prior to the selection of the textures, we performed an online survey asking a separate group of participants ( $N=54, M_{\text {age }}=25$, 46 women) what tactile sensations or textures might elicit happiness, fear, disgust, anger, surprise, shame, interest, contempt, tenderness and sadness in them. The highest-frequency answers were used to select textures for the main study. The following materials were selected: natural silk, velvet, rabbit fur (these textures potentially represented the emotion of happiness, based on the online survey in which participants indicated that something fluffy and soft can elicit happiness in them); a piece of an acupressure mat, embedded with hard plastic disks containing protruding spikes (for fear, which can be elicited by something sharp and hard); toy slime (slimy viscous liquid polymer), plasticine, clay (for disgust, elicited by something slimy and sticky); sandpaper, sponge (for anger, elicited by tough and rough textures); glass pebbles (rounded pebbles), glass seashells (for surprise, elicited by something convex and protuberant); polished marble, granite, glass (for sadness, elicited by something smooth, polished and hard). Wooden block (unpolished), wood (spruce, polished), rubber, leather, concrete, brick and tile were included as potentially neutral textures (see Figure 1). All textures varied in smoothness and hardness, according to the two main dimensions of touch experience (see Hollins et al., 1993; Picard et al., 2003; Yoshida, 1968).

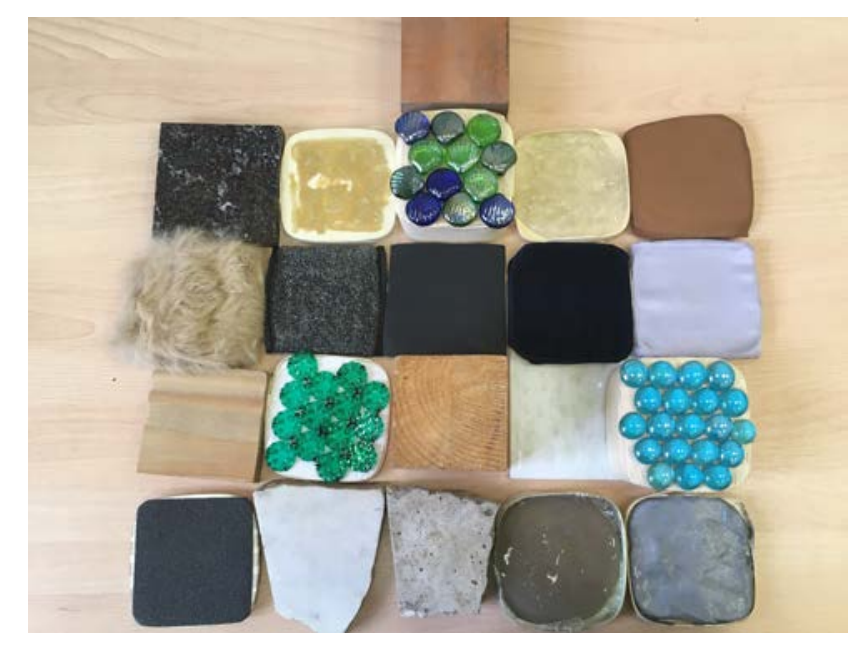


Figure 1. Twenty-one items with different textures used in the study (from left to right: brick, granite, glass, glass seashells, plasticine, leather, fur, sponge, rubber, velvet, silk, wood (spruce), acupressure mat, wooden block, tile, glass pebbles, sandpaper, marble, concrete, toy slime, clay).

\subsection{Procedure}

The experiment was performed individually in a laboratory room in daylight. Participants were seated in front of a table resting their right arm (if right-handed) on it. The experimenter was seated at a distance of $50 \mathrm{~cm}$ to the right to deliver the tactile stimulation. Participants wore an eye mask and thus could not see the textures they touched during the entire task, in order to eliminate visual cues. The experimenter placed a texture block $(9 \times 9 \mathrm{~cm})$ on the table in front of the participant and instructed him/her to touch it with their fingers and palm. Soft textures (fur, silk, etc.) were previously applied to the wooden blocks. To avoid any possible effect of sequences, the textures were presented in random order. During the touch, participants were asked to rate each texture using six evaluative scales from 0 (not at all associated with an emotion) to 5 (very much associated with an emotion). The experimenter named an emotion (e.g., surprise) and recorded the verbal score given by the participant when asked how much the texture is associated with the named emotion. The value 1 (weakly associated with the emotion) was considered the baseline for emotion association. Each texture was presented once. The experimental session lasted about 30 minutes.

After evaluating all 21 materials, participants were asked to complete the Toronto Alexithymia Scale (TAS-26; Taylor et al., 1985), translated and validated on a Russian population (Eres'ko et al., 2005). The TAS-26 is a self-report scale that measures alexithymia as a trait related to an inability to describe emotions to others and to distinguish between physical sensations and feelings. It includes 26 statements evaluated on a five-point Likert scale ( 1 - strongly disagree, 5 - strongly agree). A total TAS score greater or equal to 62 is considered to reflect a low level of alexithymia; a total score greater or equal to 74 is considered to indicate a high level of alexithymia; a total score between 63 
and 73 is considered inconclusive (Eres'ko et al., 2005; Taylor et al., 2003). Sixteen participants did not complete the TAS and were excluded from the analysis of alexithymia effects on emotion intensity.

\subsection{Statistical analysis}

The data for texture evaluation and TAS scores were both tested for normality using the onesample Kolmogorov-Smirnov test. Although the TAS scores were distributed normally, the texture evaluation data were not (Kolmogorov-Smirnov test, $p<.05$ ); therefore, we used non-parametric statistics to analyze the data. Overall, we performed four separate analyses to test our hypotheses.

To investigate whether there were any differences between the textures on each emotional scale, we used the one-way non-parametric analysis of variance (Friedman test). Six separate tests were performed, comparing the ratings of all textures on each one of the six emotions. In order to take into account multiple comparisons, only the p-values lower than .008 were considered as significant (Bonferroni correction).

To investigate which textures were significantly associated with each of the six emotions, the following analysis strategy was used. A texture was considered to be associated with a specific emotion only if the average intensity of the association was significantly higher than 1 (a significant Wilcoxon rank-sum test with a $p$-value $<$.001). Otherwise, the texture was not considered to be associated with this emotion. Only textures significantly associated with at least one of the six basic emotions were used in further analysis.

To investigate the differences between the intensity ratings of the six emotions across all textures, the Friedman analysis of variance was used. To further investigate these differences, Dunn's pairwise post hoc tests with Bonferroni correction were conducted. 
Finally, we investigated how alexithymia is related to the intensity ratings of the basic emotions. An alexithymia score was calculated for each participant and entered as a variable in the correlation analysis. We calculated the correlations between alexithymia scores and the intensity of each of the six basic emotions (evaluations of the textures as associated with fear, anger, happiness, disgust, sadness and surprise). Because the data were not distributed normally, we used nonparametric correlations and reported the results of two-tailed Spearman's correlation coefficient tests. Statistical analysis was performed in SPSS (IBM, Armonk, Version 25.0, 2017) and R (R Core Team, 2013).

\section{Results}

\subsection{Effects of emotional evaluation on textures}

Friedman tests comparing the average levels of emotion intensity across textures were significant for each emotional scale: happiness $\left(\chi(20)^{2}=441.58, p<.0001\right)$, fear $\left(\chi(20)^{2}=343.86, p\right.$ $<.0001)$, disgust $\left(\chi(20)^{2}=510.26, p<.0001\right)$, anger $\left(\chi(20)^{2}=338.36, p<.0001\right)$, surprise $\left(\chi(20)^{2}=\right.$ 395.16, $p<.0001)$, sadness $\left(\chi(20)^{2}=120.19, p<.0001\right)$. That means that there are differences between the textures in terms of how they are emotionally evaluated. We next investigated which textures were significantly associated with each of the six emotions.

Thirteen textures were associated with happiness; eight textures were associated with fear; six textures were associated with disgust; four textures were associated with anger; nine textures were associated with sadness; and all textures were associated with surprise. Overall, all textures were associated with some emotion. Some textures were associated with specific emotions (e.g., marble is mainly associated with sadness, fur is mainly associated with happiness), while others were associated with several emotions (e.g., a sponge is associated with fear, disgust and anger). Table 1 shows the 
medians and interquartile ranges for the textures associated with six emotions significantly higher than 1 (a significant Wilcoxon rank-sum test with a $p$-value $<.001$ ).

Table 1. Medians and interquartile ranges of emotional ratings of textures

\begin{tabular}{|c|c|c|c|c|c|c|}
\hline & Happiness & Fear & Disgust & Anger & Surprise & Sadness \\
\hline Acupressure mat & - & $3(2)$ & $2(3.75)$ & $3(2.75)$ & $3(3)$ & - \\
\hline Plasticine & - & $2(3)$ & $4(2)$ & $1(2.75)$ & $3(2)$ & - \\
\hline Fur & $4(2)$ & - & - & - & $3(2)$ & - \\
\hline Sandpaper & - & $2(2)$ & $2(4)$ & $2(1)$ & $2(2)$ & - \\
\hline Velvet & $4(2)$ & - & - & - & $2(2)$ & - \\
\hline Rubber & $2(3)$ & - & - & - & $2(3)$ & - \\
\hline Toy slime & $1(3)$ & $2(4)$ & $4(3)$ & - & $4(2)$ & - \\
\hline Leather & $2(3)$ & - & - & - & $2(2)$ & - \\
\hline Silk & $3(2)$ & - & - & - & $2(2)$ & $1(3)$ \\
\hline Clay & - & $1(2)$ & $2(2)$ & - & $3(1.75)$ & - \\
\hline Glass & $2(3)$ & $1(3)$ & - & - & $2(2)$ & $2(3)$ \\
\hline Wood & $2(3)$ & - & - & - & $2(3)$ & $1(3)$ \\
\hline Wooden block & $2(2)$ & - & - & - & $1(3)$ & $1(3)$ \\
\hline Marble & - & - & - & - & $1(2)$ & $2(3)$ \\
\hline Concrete & $2(3)$ & - & - & - & $1(3)$ & $1(3)$ \\
\hline Brick & - & - & - & - & $1.5(3)$ & $2(3)$ \\
\hline Tile & $2(3)$ & - & - & - & $2(2.75)$ & $1(3)$ \\
\hline
\end{tabular}




\begin{tabular}{lcccccc} 
Glass pebbles & $3(2)$ & - & - & - & $3(2)$ & - \\
Granite & - & $2(3)$ & - & - & $1.5(3)$ & $2(4)$ \\
Glass seashells & $2(3)$ & - & - & - & $3(2.75)$ & - \\
Sponge & - & $3(2)$ & $2(3)$ & $3(4)$ & $3(2.75)$ & - \\
Total & $2(3)$ & $1(3)$ & $1(3)$ & $1(2)$ & $2(3)$ & $1(3)$ \\
\hline
\end{tabular}

Note. Interquartile ranges are designated in round brackets. The scale ranged from 0 (not at all associated with the emotion) to 5 (very much associated with the emotion).

\subsection{Effects of emotion type on emotion intensity}

There was a significant difference between intensity ratings of the six emotions: $\left(\chi(5)^{2}=\right.$ 49.53, $p<.001$ ). Dunn's pairwise tests revealed differences between sadness intensity and the intensity of the other five emotions ( $p<.05$, adjusted using the Bonferroni correction): sadness was less intense compared to anger $(p<.001)$, fear $(p=.017)$, disgust $(p<.001)$, surprise $(p=.001)$ and happiness ( $p=.001$ ); see Figure 2. There was also a difference between the intensity of disgust and fear $(p<.001$, adjusted using the Bonferroni correction): the intensity of disgust was greater compared to the intensity of fear (see Figure 2).

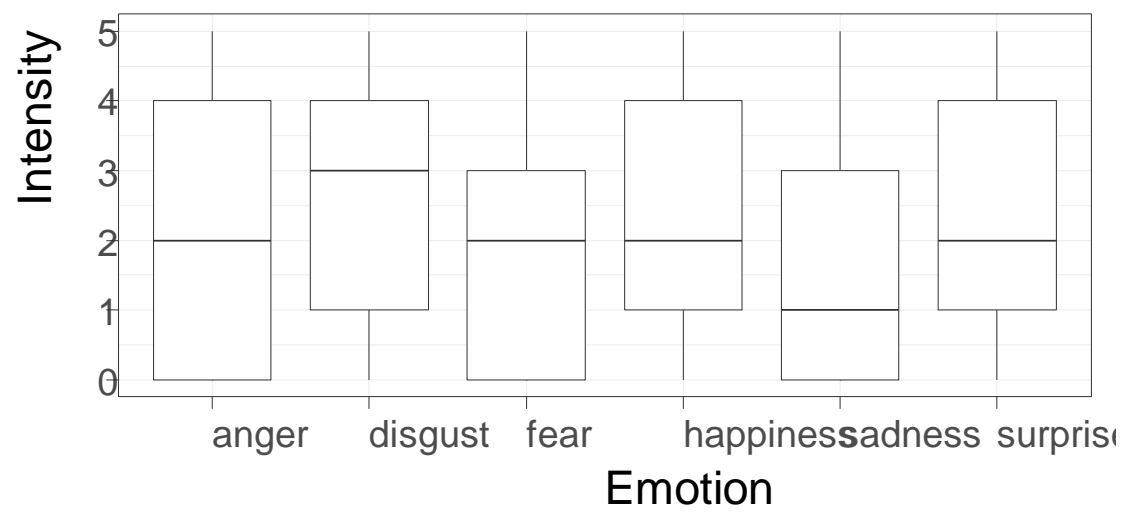


Figure 2. Medians, interquartile ranges and full ranges of intensity ratings of six emotions associated with touching textures.

\subsection{Effects of alexithymia on emotion intensity}

The mean TAS level across participants was $58.14(S D=9.77)$. Spearman's correlation analysis between TAS level and emotion intensity revealed that alexithymia correlated significantly with disgust intensity ( $r h o=.109, p=.020)$, anger intensity $(r h o=.107, p=.043)$ and sadness intensity $(r h o=.094, p=.007)$. No significant correlations were found with surprise intensity ( $r h o=.009, \mathrm{p}=$ $.688)$, happiness intensity ( $r h o=.044, p=.135)$ or fear intensity ( $r h o=.046, p=.217)$. Higher levels of alexithymia were therefore associated with higher intensities of disgust, anger and sadness.

\section{Discussion}

The present study investigated whether different textures are associated with any of six basic emotions. In an online survey, participants named tactile sensations and textures that can elicit particular emotions in them. Based on the most frequently named sensations, we selected 21 textures that can potentially be associated with basic emotions. In a lab-based experiment, a separate group of participants touched these textures and evaluated them on six emotional scales. As a result, we revealed a set of textures associated with six basic emotions. The emotion of surprise differed from the other emotions, because all textures were associated with it. This might be related to the fact that the participants did not see the textures and did not have any prior visual knowledge about them.

The associations between textures and emotions can partly be explained by the role of temperature and softness/roughness in pleasure. The textures varied in temperature and this fact might have an impact on associations between them and emotions. Concerning roughness, previous studies showed that soft and gentle touch sensations are associated with pleasantness, while roughness is correlated with unpleasantness (Major, 1895; Zampini et al., 2003), Indeed, our soft materials, such 
as fur, velvet, silk and leather, were associated with the positive emotion of happiness; rough materials such as sandpaper, an acupressure mat and a wire sponge were associated with negative emotions (fear, anger and disgust). However, participants’ softness/roughness and temperature ratings would need to be studied further to confirm these suggestions.

However, softness and roughness cannot fully explain the associations between textures and basic emotions: toy slime and plasticine are not rough, but they were associated with fear and disgust; glass pebbles are not soft, but they were associated with happiness; smooth rather than rough materials (e.g., silk, marble, granite) were associated with sadness. Another possible explanation is that textures might be related to emotions because of the objects associated with these textures (e.g., marble, concrete and granite are associated with monuments, which are often dedicated to dead people and found at cemeteries, and therefore are associated with sadness). This supposition can be in line with the ecological valence theory, which postulates that associations with objects explain color preferences (Palmer \& Schloss, 2010). However, the present study did not aim to explain the nature of affective associations with textures, and in particular whether they are mediated by conceptual knowledge and categorization; future studies may aim to do that. The main aim of the present study was to provide evidence that while touching different textures, people associate distinct emotions with them.

In addition, the study found that there are differences in the intensity of emotional associations with textures. This might indicate that people recognize some emotions (e.g., disgust) via touch more readily compared to other emotions (e.g., sadness).

Another finding concerns the role of individual differences in alexithymia in associating emotions with textures. A higher level of alexithymia was related to a higher perceived intensity of disgust, anger and sadness. This result is consistent with previously found differences in negative 
emotion perception (particularly, fear and anger) among people with high and low levels of alexithymia (Prkachin, Casey \& Prkachin, 2008; Scarpazza et al., 2014). However, it was supposed that the perceived intensity of emotions is lower among people with alexithymia, while our study found the contrary. Our finding can be explained from the perspective of hyperarousal theory, which supposes that the mechanism of alexithymia is associated with higher emotional reactivity (Martin \& Pihl, 1986). Some empirical studies found support for this hypothesis in the visual and olfactory perception of emotions (Eastabrook, Lanteigne \& Hollenstein, 2013; Lombion et al., 2010). Our study supports the hyperarousal theory in the haptic modality. Moreover, Hosoi and colleagues (2010) showed that perceived pain intensity is higher among people with higher levels of alexithymia. This might support the idea of enhanced perception of negative affective states among people with alexithymia. To our knowledge, this is the first study investigating the role of alexithymia in associating emotions with textures.

There are some important limitations in the present study. Firstly, the velocity at which the palm and fingers contacted the textures and the indenting force were not controlled. It is possible that velocity and indenting force impacted the emotional evaluation of textures (Cascio et al., 2008; Essick et al., 1999). Secondly, while touching the textures, participants did not wear earplugs, and thus auditory stimulation was not eliminated. It is possible that some auditory clues might have impacted the textures' evaluation. For example, rough textures may generate rough sounds when stroked and these sounds may evoke emotional associations of their own. Finally, only 22 males participated in this study, which limited us in investigating any gender effects on emotional associations with textures. Studies show that there are gender differences in emotion intensity perception (Biele \& Grabowska, 2006; Hoffmann et al., 2010; Mandal \& Palchoudhury 1985; Rotter \& Rotter 1988; Wagner et al., 1986). Future research may address this issue in haptic emotional perception. It would 
also be interesting to further investigate whether textures might be positively or negatively valenced depending on whether participants could see them or at least know what it was that they were touching.

To conclude, the study extends the literature on the perception of emotion in three ways. First, it provides evidence that while touching different textures, people perceive not only their valence, but also distinct emotions associated with them. Secondly, it identified the textures significantly associated with six basic emotions, which can be further used to investigate the perception of emotions via touch. Thirdly, it showed that individual differences in alexithymia levels are related to the intensity of negative emotional associations with some textures.

From a basic perspective, the set of textures we developed, together with their emotional ratings, might be of interest to researchers who investigate emotional responsiveness to textures, and want to use textures associated with basic emotions or neutral textures. While there are several sets of normative emotional stimuli for experimental investigations of emotion in vision and audition (Bradley \& Lang, 2007; Lang, Bradley \& Cuthbert, 2008), there is a lack of such sets in haptics. Although the use of exactly the same physical stimuli by other haptics researchers is not as easy as the use of digital images in the visual modality or digital sounds in the auditory modality, the description of our dataset and their emotional ratings might be useful in conducting further studies in the haptic modality. From an applied perspective, these findings can be used in product design in order to produce particular expectations among customers (Gallace \& Spence, 2014; Spence, 2014; Spence \& Gallace, 2011).

Acknowledgments. The authors thank Andrey Kiselnikov for his kind assistance in research organization. The authors thank Oksana Zinchenko for her kind assistance in the recruitment of participants.

Funding. This work was supported by Russian Foundation for Basic Research Grant \#18-013-01221.

Conflict of interest. The authors declare no conflict of interest. 
Data access statement. Data directly supporting the publication can be found at https://osf.io/4xuem/

\section{References}

Ackerley, R., Carlsson, I., Wester, H., Olausson, H., \& Backlund Wasling, H. (2014). Touch perceptions across skin sites: differences between sensitivity, direction discrimination and pleasantness. Frontiers in Behavioral Neuroscience, 8, 54.

https://doi.org/10.3389/fnbeh.2014.00054

Biele, C., \& Grabowska, A. (2006). Sex differences in perception of emotion intensity in dynamic and static facial expressions. Experimental Brain Research, 171(1), 1-6. https://doi.org/10.1007/s00221-005-0254-0

Bradley, M. M. \& Lang, P. J. (2007). The International Affective Digitized Sounds (2nd Edition; IADS-2): Affective ratings of sounds and instruction manual. Technical report B-3. University of Florida, Gainesville, Fl.

Cascio, C., McGlone, F., Folger, S., Tannan, V., Baranek, G., Pelphrey, K. A., \& Essick, G. (2008). Tactile perception in adults with autism: a multidimensional psychophysical study. Journal of Autism and Developmental Disorders, 38(1), 127-137. https://doi.org/10.1007/s10803-007-0370-8

Cecchetto, C., Rumiati, R. I., \& Aiello, M. (2017). Alexithymia and emotional reactions to odors. Scientific Reports, 7(1), 14097. https://doi.org/10.1038/s41598-017-14404-X

Chapman, C. E. (1994) Active versus passive touch: factors influencing the transmission of somatosensory signals to primary somatosensory cortex. Canadian Journal of Physiological Pharmacology, 72, 558-570.

Croy, I., Luong, A., Triscoli, C., Hofmann, E., Olausson, H., \& Sailer, U. (2016). Interpersonal stroking touch is targeted to C tactile afferent activation. Behavioural Brain Research, 297, 3740. https://doi.org/10.1016/j.bbr.2015.09.038

Du, S., \& Martinez, A. M. (2011). The resolution of facial expressions of emotion. Journal of Vision, 11(13), 24. https://doi.org/10.1167/11.13.24

Eastabrook, J. M., Lanteigne, D. M., \& Hollenstein, T. (2013). Decoupling between physiological, self-reported, and expressed emotional responses in alexithymia. Personality and Individual Differences, 55(8), 978-982. https://doi.org/10.1016/j.paid.2013.08.001

Eibl-Eibesfeldt, I. (1989). Human ethology. Hawthorne, NY: Aldine de Gruyter.

Ekman, P. (1993). Facial expression and emotion. The American Psychologist, 48(4), 384-392.

Ekman, G., Hosman, J., Lindstrom, B., 1965. Roughness, smoothness, and preference: a study of quantitative relations in individual subjects. Journal of Experimental Psychology 70, 18-26.

Eres'ko D.B., Isurina G.L., Kajdanovskaya E.V., Karvasarskij B.D., Karpova Eh.B., Korepanova T.G., Krylova G.S., Tarhan A.U., Chekhlatyj E.I., Shifrin, V.B. (2005). Alexithymia and its diagnostics among people with psychosomatic psychosomatic disorders. Saint-Petersburg: 
Sankt-Peterburgskij nauchno-issledovatel'skij psihonevrologicheskij institut im. V. M. Bekhtereva.

Essick, G. K., James, A., \& McGlone, F. P. (1999). Psychophysical assessment of the affective components of non-painful touch. Neuroreport, 10(10), 2083-2087.

Essick, G. K., McGlone, F., Dancer, C., Fabricant, D., Ragin, Y., Phillips, N., ... Guest, S. (2010). Quantitative assessment of pleasant touch. Neuroscience and Biobehavioral Reviews, 34(2), 192-203. https://doi.org/10.1016/j.neubiorev.2009.02.003

Etzi, R., Spence, C., Zampini, M., \& Gallace, A. (2016). When sandpaper is "Kiki” and satin is "Bouba": an exploration of the associations between words, emotional states, and the tactile attributes of everyday materials. Multisensory Research, 29(1-3), 133-155.

Etzi, R., Spence, C., \& Gallace, A. (2014). Textures that we like to touch: an experimental study of aesthetic preferences for tactile stimuli. Consciousness and Cognition, 29, 178-188. https://doi.org/10.1016/j.concog.2014.08.011

Feldman, R., Gordon, I., Schneiderman, I., Weisman, O., \& Zagoory-Sharon, O. (2010). Natural variations in maternal and paternal care are associated with systematic changes in oxytocin following parent-infant contact. Psychoneuroendocrinology, 35(8), 1133-1141. https://doi.org/10.1016/j.psyneuen.2010.01.013

Fernandez, E., \& Turk, D. C. (1992). Sensory and affective components of pain: separation and synthesis. Psychological Bulletin, 112(2), 205-217.

Field, T. (2010). Touch for socioemotional and physical well-being: A review. Developmental Review, 30(4), 367-383. https://doi.org/10.1016/j.dr.2011.01.001

Gallace, A., \& Spence, C. (2014). In Touch with the future: the sense of touch from cognitive neuroscience to virtual reality. Oxford University Press, Oxford, UK.

Gallace, A., \& Spence, C. (2010). The science of interpersonal touch: an overview. Neuroscience and Biobehavioral Reviews, 34(2), 246259. https://doi.org/10.1016/j.neubiorev.2008.10.004

de Gelder, B. L., \& Vroomen, J. (2000). The perception of emotions by ear and by eye. Cognition \& Emotion, 14(3), 289-311. https://doi.org/10.1080/026999300378824

Hamann, S., \& Canli, T. (2004). Individual differences in emotion processing. Current Opinion in Neurobiology, 14(2), 233-238. https://doi.org/10.1016/j.conb.2004.03.010

Hertenstein, M. J., Holmes, R., McCullough, M., \& Keltner, D. (2009). The communication of emotion via touch. Emotion, 9(4), 566-573. https://doi.org/10.1037/a0016108

Hertenstein, M. J., Keltner, D., App, B., Bulleit, B. A., \& Jaskolka, A. R. (2006). Touch communicates distinct emotions. Emotion, 6(3), 528-533. https://doi.org/10.1037/1528$\underline{3542.6 .3 .528}$ 
Hoffmann, H., Kessler, H., Eppel, T., Rukavina, S., \& Traue, H. C. (2010). Expression intensity, gender and facial emotion recognition: Women recognize only subtle facial emotions better than men. Acta Psychologica, 135(3), 278-283. https://doi.org/10.1016/j.actpsy.2010.07.012

Hollins, M., Faldowski, R., Rao, S., \& Young, F. (1993). Perceptual dimensions of tactile surface texture: a multidimensional scaling analysis. Perception \& Psychophysics, 54(6), 697705.

Hosoi, M., Molton, I. R., Jensen, M. P., Ehde, D. M., Amtmann, S., O’Brien, S., ... Kubo, C. (2010). Relationships among alexithymia and pain intensity, pain interference, and vitality in persons with neuromuscular disease: considering the effect of negative affectivity. Pain, 149(2), 273-277. https://doi.org/10.1016/j.pain.2010.02.012

IBM Corp. Released 2017. IBM SPSS Statistics for Windows, Version 25.0. Armonk, NY: IBM Corp.

Iosifyan, M., Korolkova, O., \& Vlasov, I. (2017). Emotional and semantic associations between cinematographic aesthetics and haptic perception. Multisensory Research, 30, 783-798.

Jenkins, J. L. (1986). Physiological effects of petting a companion animal, physiological effects of petting a companion animal. Psychological Reports, 58(1), 21-22.

https://doi.org/10.2466/pr0.1986.58.1.21

Kirsch, L. P., Krahé, C., Blom, N., Crucianelli, L., Moro, V., Jenkinson, P. M., \& Fotopoulou, A. (2018). Reading the mind in the touch: Neurophysiological specificity in the communication of emotions by touch. Neuropsychologia, 116, 136-149.

Lang, P. J., Bradley, M. M., \& Cuthbert, B. N. (2008). International affective picture system (IAPS): Affective ratings of pictures and instruction manual. Technical Report A-8. University of Florida, Gainesville, FL.

Lane, R. D., Sechrest, L., Reidel, R., Weldon, V., Kaszniak, A., \& Schwartz, G. E. (1996). Impaired verbal and nonverbal emotion recognition in alexithymia. Psychosomatic Medicine, 58(3), 203-210.

Lass-Hennemann, J., Peyk, P., Streb, M., Holz, E., \& Michael, T. (2014). Presence of a dog reduces subjective but not physiological stress responses to an analog trauma. Frontiers in Psychology, 5. https://doi.org/10.3389/fpsyg.2014.01010

Lederman, S. J., Klatzky, R. L. (1987). Hand movements: a window into haptic object recognition. Cognitive Psychology, 19(3): 342-368. doi:10.1016/0010-0285(87)90008-9.

Löken, L. S., Wessberg, J., Morrison, I., McGlone, F., \& Olausson, H. (2009). Coding of pleasant touch by unmyelinated afferents in humans. Nature Neuroscience, 12(5), 547-548. https://doi.org/10.1038/nn.2312

Lombion, S., Bechetoille, B., Nezelof, S., \& Millot, J.-L. (2010). Odor perception in alexithymic patients. Psychiatry Research, 177(1-2), 135-138.

https://doi.org/10.1016/j.psychres.2009.01.018 
Major, D. R. (1895). On the affective tone of simple sense-impressions. American Journal of Psychology, 7, 57-77.

Mandal, M. K., \& Palchoudhury, S. (1985). Responses to facial expression of emotion in depression. Psychological Reports, 56(2), 653-654. https://doi.org/10.2466/pr0.1985.56.2.653

Martin, J.B. \& Pihl, R.O. (1986). Influence of alexithymic characteristics on physiological and subjective stress responses in normal individuals. Psychotherapy and Psychosomatics, 45, 6677.

Martins, A. T., Ros, A., Valério, L., \& Faísca, L. (2017). Basic emotion recognition according to clinical personality traits. Current Psychology, 36, 1-11. https://doi.org/10.1007/s12144-0179661-1

McDonald, P. W., \& Prkachin, K. M. (1990). The expression and perception of facial emotion in alexithymia: a pilot study. Psychosomatic Medicine, 52(2), 199-210.

Mohn, C., Argstatter, H., \& Wilker, F.-W. (2011). Perception of six basic emotions in music. Psychology of Music, 39(4), 503-517. https://doi.org/10.1177/0305735610378183

Palmer, S. E., \& Schloss, K. B. (2010). An ecological valence theory of human color preference. Proceedings of the National Academy of Sciences, 107(19), 8877-8882. https://doi.org/10.1073/pnas.0906172107

Parker, P. D., Prkachin, K. M., \& Prkachin, G. C. (2005). Processing of facial expressions of negative emotion in alexithymia: the influence of temporal constraint. Journal of Personality, 73(4), 1087-1107. https://doi.org/10.1111/j.1467-6494.2005.00339.x

Peled-Avron, L., Goldstein, P., Yellinek, S., Weissman-Fogel, I., \& Shamay-Tsoory, S. G. (2017). Empathy during consoling touch is modulated by mu-rhythm: An EEG study. Neuropsychologia. https://doi.org/10.1016/j.neuropsychologia.2017.04.026

Picard, D., Dacremont, C., Valentin, D., \& Giboreau, A. (2003). Perceptual dimensions of tactile textures. Acta Psychologica, 114(2), 165-184.

Prkachin, G. C., Casey, C., \& Prkachin, K. M. (2009). Alexithymia and perception of facial expressions of emotion. Personality and Individual Differences, 46(4), 412-417.

https://doi.org/10.1016/j.paid.2008.11.010

R Core Team (2013). R: A language and environment for statistical computing. R Foundation for Statistical Computing, Vienna, Austria. URL http://www.R-project.org/.

Rotter, N. G., \& Rotter, G. S. (1988). Sex differences in the encoding and decoding of negative facial emotions. Journal of Nonverbal Behavior, 12(2), 139-148.

https://doi.org/10.1007/BF00986931

Sauter, D. A., Eisner, F., Ekman, P., \& Scott, S. K. (2010). Cross-cultural recognition of basic emotions through nonverbal emotional vocalizations. Proceedings of the National Academy of Sciences of the United States of America, 107(6), 2408-2412.

https://doi.org/10.1073/pnas.0908239106

Scarpazza, C., di Pellegrino, G., \& Làdavas, E. (2014). Emotional modulation of touch in alexithymia. Emotion, 14(3), 602-610. 
Simoes-Franklin, C., Whitaker, T. A., \& Newell, F. N. (2011). Active and passive touch differentially activate somatosensory cortex in texture perception. Human Brain Mapping, 32(7), 1067-1080. https://doi.org/10.1002/hbm.21091

Spence, C. (2014). Assessing the influence of shape and sound symbolism on the consumer's response to chocolate. New Food, 17, 59-62.

Spence, C. \& Gallace, A. (2011). Multisensory design: reaching out to touch the consumer. Psychology and Marketing, 28, 267-308.

Stack, D. M., \& Muir, D. W. (1990). Tactile stimulation as a component of social interchange: New interpretations for the still-face effect. British Journal of Developmental Psychology, 8(2), 131-145. https://doi.org/10.1111/j.2044-835X.1990.tb00828.x

Van den Stock, J., Righart, R., \& de Gelder, B. (2007). Body expressions influence recognition of emotions in the face and voice. Emotion, 7(3), 487-494. https://doi.org/10.1037/15283542.7.3.487

Suvilehto, J. T., Glerean, E., Dunbar, R. I. M., Hari, R., \& Nummenmaa, L. (2015). Topography of social touching depends on emotional bonds between humans. Proceedings of the National Academy of Sciences, 112(45), 13811-13816. https://doi.org/10.1073/pnas.1519231112

Taylor, G. J., Ryan, D., \& Bagby, R. M. (1985). Toward the development of a new self-report alexithymia scale. Psychotherapy and Psychosomatics, 44(4), 191-199.

https://doi.org/10.1159/000287912

Taylor, G.J., Bagby, R.M., \& Parker, J.D.A. (1997). Disorders of affect regulation. Cambridge, United Kingdom: Cambridge University Press.

Taylor, G. J., Bagby, R. M., \& Parker, J. D. A. (2003). Disorders of affect regulation: alexithymia in medical and psychiatry illness. Cambridge, United Kingdom: Cambridge University Press.

Verrillo, R. T., Bolanowski, S. J., \& McGlone, F. P. (1999). Subjective magnitude of tactile roughness. Somatosensory and Motor Research, 16, 352-360.

Wagner, H. L., MacDonald, C. J., \& Manstead, A. S. (1986). Communication of individual emotions by spontaneous facial expressions. Journal of Personality and Social Psychology, 50(4), 737-743. https://doi.org/10.1037/0022-3514.50.4.737

Yoshida, M. (1968). Dimensions of tactual impressions (1). Japanese Psychological Research, 10, 123-137.

Zampini, M., Guest, S., \& Spence, C. (2003). The role of auditory cues in modulating the perception of electric toothbrushes. Journal of Dental Research, 82, 929-932. 